\title{
Kids-InteracTOOL: an interactive tool to promote parent-child interaction for the development of educational and social skills in pandemic times
}

\author{
Jessica Cordova-Rangel, Moisés Ramsés Jauregui-Sanchez, Martha Isabel \\ Chávez, Karina Caro
}

Published: 30 November 2021

\begin{abstract}
Children ${ }^{1}$ have increased their use of technological devices due to the COVID-19 pandemic, which implies that they have a better domain of this technology. However, with the pandemic, they have been affected educationally and socially by changes in the format they take their classes and by the lack of social interaction. The people with whom they interact immediately are their parents or guardians, who sometimes do not have the time or knowledge to provide them with tools to develop their skills. Therefore, this project proposes the design of a tool, using the Design Thinking methodology, based on a videogame that supports the development of educational and social skills of children and allows collaboration with their parents or guardians.
\end{abstract}

\section{Keywords:}

User-centered Design; Design Thinking; Videogame; Social skills; Learning skills.

\section{Introducción}

El confinamiento por COVID-19 representó un aumento en el número de usuarios de Internet en comparación con los últimos cinco años. Aunque esto no representó una reducción en la brecha digital y de acceso a la tecnología y al internet [1].

A nivel educativo, la pandemia implicó la transformación de las clases presenciales a un formato a distancia (o en línea), lo que representó cambios en el contenido de clases, restricciones de acceso por falta de recursos materiales como dispositivos tecnológicos, conectividad con internet, así como complicaciones a nivel aprendizaje y manejo de las herramientas. De tal manera que el aprendizaje de los estudiantes se ha visto impactado no solo a nivel académico, sino también a nivel social, en el que se ha visto un aumento considerable en el uso de tecnología [3] y una reducción notable en las interacciones interpersonales.

Cordova-Rangel, Jessica., Jauregui-Sanchez, Moisés Ramsés., Chávez, Martha Isabel., Caro, Karina.

Universidad Autónoma de Baja California (UABC)

Ensenada, Mexico

jessica.cordova.rangel@uabc.edu.mx,moises.jauregui@uabc.edu.mx, chabely@uabc.edu.mx, karina.caro@uabc.edu.mx

\footnotetext{
${ }^{1}$ Por simplicidad de la lectura, en este documento se hace referencia a los niños y niñas, solo como niños.
}

Considerando que aproximadamente $55 \%$ de los niños son cuidados por sus abuelos mientras sus padres de familia o tutores trabajan [7], se deben buscar alternativas para que los niños cuenten con herramientas tecnológicas que les permitan desarrollar sus habilidades de lecto-escritura, lógico-matemáticas y habilidades sociales, con la guía mínima de los adultos (teniendo en cuenta que estos en ocasiones no cuentan con los recursos de tiempo y conocimiento para orientarlos), pero con una participación relevante en su aprendizaje, y esto se ha visto favorecido con el uso de videojuegos educativos [6] debido a que logran generar un interés para realizar las actividades. En este proyecto se propone un videojuego para apoyar las habilidades educativas y sociales de niños en etapa escolar básica (preescolar y primaria), la cual fomenta la interacción entre los niños y sus padres/tutores.

\section{Trabajo Relacionado}

Existen diversos esfuerzos para apoyar el desarrollo de habilidades educativas y sociales de los niños mediante herramientas tecnológicas $[12,17]$. Estos generalmente incluyen un enfoque basado en juegos, dado que el "juego permite a los niños comunicar ideas y entender a los demás mediante la interacción social" [16]. Por lo que, el uso de videojuegos promueve el entretenimiento [5], la educación [15], el desarrollo de habilidades [1] y la socialización [2]. Hay una gran variedad de videojuegos para el desarrollo de habilidades sociales $[11,12,14]$, y habilidades educativas $[4,13]$. Por ejemplo, en Monkey Tales [4], un juego de matemáticas, se encontró que a los adultos no les agrada que los niños pasen mucho tiempo jugando juegos digitales, pero si van a permitir que los niños los usen, deben ser juegos educativos. Por otro lado, en Aboard the S.S.GRIN [14], un sistema inteligente de tutoría social, aun con una exposición limitada de juego, los niños lograron desarrollar sus habilidades sociales. EmoFindAR [14] es un juego móvil de realidad aumentada multijugador que busca fomentar la socialización y la inteligencia emocional en niños de primaria. Este trabajo concluye que un juego multijugador de realidad aumentada desencadena emociones positivas. Estos proyectos tienen algunas características en común, como el uso de recompensas, historias de juego, repetición de actividades, entre otros. No obstante, ninguno de los proyectos fomenta la interacción entre el adulto y el niño, dado que las actividades son exclusivas para que el niño participe; 
así, existen áreas de oportunidad para videojuegos que favorezcan el aprendizaje y entretenimiento; así como la interacción entre el adulto y el niño mediante actividades guiadas.

\section{Metodología}

La metodología empleada en este proyecto es la metodología centrada en el usuario Design Thinking, la cual está compuesta de cinco etapas: Empatizar, Definir, Idear, Prototipar y Evaluar.

\subsection{Empatizar}

Esta fase implica entender las características y las necesidades de los usuarios para los cuales se va a diseñar, y así profundizar en el contexto de la problemática. Se utilizaron técnicas de recolección de datos como la observación no participativa y observación directa participativa a niños en edad escolar mientras tomaban alguna de sus clases de manera virtual y se realizaron entrevistas semiestructuradas a los padres de familia o tutores de estos niños. Para esto, se diseñó un protocolo de entrevista y se solicitó un consentimiento informado firmado por parte del adulto (padre de familia o tutor). Se realizaron dos sesiones de observación a los niños bajo la autorización de los padres en su hogar. En la Tabla 1 se muestra la duración de las entrevistas y las observaciones, si bien una de las observaciones fue participativa esto se debió a que el niño tenía previa conexión con la investigadora y en todo momento quiso interactuar con ella.

Tabla 1. Resumen de los métodos de recolección de datos.

\begin{tabular}{|c|c|c|c|}
\hline \# & Tipo & $\begin{array}{l}\text { Detalle de los } \\
\text { participantes }\end{array}$ & $\begin{array}{l}\text { Duración } \\
\text { hrs:mm:ss }\end{array}$ \\
\hline 1 & Entrevista & $\begin{array}{l}\text { Madre } 41 \text { años, } \\
\text { profesionista }\end{array}$ & $00: 29: 50(\mathrm{n}=1)$ \\
\hline 2 & Entrevista & $\begin{array}{l}\text { Madre } 38 \text { años, } \\
\text { ama casa }\end{array}$ & $00: 22: 14(\mathrm{n}=1)$ \\
\hline 3 & Entrevista & $\begin{array}{l}\text { Madre } 43 \text { años, } \\
\text { ama casa }\end{array}$ & $00: 23: 10(\mathrm{n}=1)$ \\
\hline 4 & $\begin{array}{l}\text { Observación } \\
\text { directa } \\
\text { participativa }\end{array}$ & Niño 5 años & $01: 61: 00(\mathrm{n}=2)$ \\
\hline 5 & $\begin{array}{l}\text { Observación no } \\
\text { participativa }\end{array}$ & Niño 5 años & $01: 23: 00(\mathrm{n}=2)$ \\
\hline 6 & $\begin{array}{l}\text { Observación no } \\
\text { participativa }\end{array}$ & Niño 7 años & $01: 30: 00(\mathrm{n}=2)$ \\
\hline
\end{tabular}

\subsection{Definir}

Durante esta fase, el grupo de trabajo analizó la información recolectada en la fase anterior. El objetivo de esta fase fue delimitar el alcance del proyecto teniendo en cuenta las prácticas actuales de niños que realizan actividades escolares de manera remota, así como conocer el papel que juegan los padres/tutores. Los métodos empleados en esta etapa fueron: codificación abierta y axial [9], las cuales son técnicas de análisis cualitativo donde se extraen códigos o conceptos relevantes de la información de las observaciones y entrevistas. Posteriormente, se realizó una sesión de interpretación para realizar un diagrama de afinidad donde los códigos o conceptos se agrupan en función de categorías, propiedades y dimensiones. Adicionalmente, se empleó la técnica de personas [8] para crear personajes ficticios que sirven para representar diferentes tipos de usuarios.

\subsection{Idear y prototipar}

En la fase de idear el propósito es generar ideas tomando en cuenta los resultados de las etapas anteriores para poder proponer soluciones a la problemática identificada. Los métodos empleados para el proceso de idear fueron lluvia de ideas (brainstorming) y de Crazy 8 's. La técnica de Crazy 8's [10] consiste en una actividad de dibujo rápido en el que cada participante de la sesión tiene que crear ocho pantallas diferentes de la herramienta en menos de 8 minutos, lo que implica un minuto por pantalla (Ver Figura con ejemplos de resultados de lluvia de ideas y Crazy 8's). Una vez concluida la fase de idear, procedió la fase de prototipar en donde se priorizaron y votaron las mejores ideas por parte del equipo de trabajo, las ideas seleccionadas se transformaron en un prototipo de baja fidelidad, el cual se construyó utilizando la herramienta de prototipado en línea Figma ${ }^{2}$.

\subsection{Evaluar}

En esta fase, el prototipo realizado con Figma se mostró a tres madres de familia y sus tres niños para explicarles la propuesta de la herramienta y recibir retroalimentación. Se utilizó el Cuestionario de Aceptación de la Tecnología (Technology Acceptance Model, TAM) para conocer la utilidad y la facilidad de uso por parte de la madre de familia. El TAM utiliza una escala 7puntos Likert. Además, se utilizó el cuestionario de experiencia de juego (Game Experience Questionnaire, GEQ) para conocer la posible experiencia de juego de los niños. El GEQ utiliza una escala de 5-puntos Likert.

\section{Principales resultados de las Etapas de Empatizar y Definir 4.1 Diagrama de Afinidad}

Entre las categorías que se identificaron en el diagrama de afinidad (Figura 1) se tienen las áreas educativas de oportunidad, características de las clases remotas, actividades de ocio, tecnología deseada y características del niño. Por ejemplo, en áreas educativas de oportunidad se encuentran las dificultades tecnológicas y las estrategias de seguimiento sobre el progreso del niño; en características de las clases remotas la frecuencia de las clases y las dinámicas que se aplican en estas. Esta afinidad por las actividades que empleen tecnologías, sumada a las características que los niños tienen en común, propicia el diseño de una herramienta enfocada en hacer avances sobre el desempeño de un niño en varias habilidades como lo son la lectoescritura, lógico-matemáticas y las habilidades sociales.

\subsection{Creación de Personas}

Se crearon dos personas, una persona niño y una persona adulta con el fin de tener presente sus intereses, características y limitaciones al momento de idear propuestas para la solución. La creación de estas personas se realizó con base en los resultados del diagrama de afinidad (Ver Figura de Personas). Un punto de vista (Point-ofview, POV) es una declaración significativa del problema, que permite idear posibles soluciones al problema de una manera orientada a objetivos. El POV captura la visión de diseño definiendo el reto correcto a abordar en la fase de Idear (Ver Figura del POV).

\footnotetext{
${ }^{2}$ https://www.figma.com/
} 


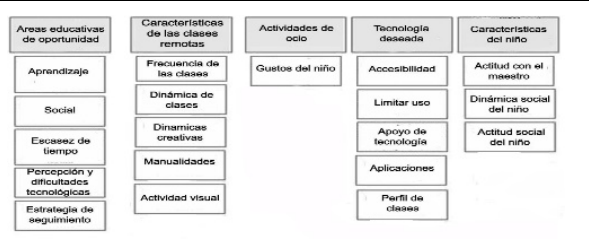

Figura 1. Características del Diagrama de Afinidad.

\section{Resultados del Proceso de Diseño}

Con base en los resultados del diagrama de afinidad, se identificaron tres temáticas principales que los padres de familia comentaron como relevantes, los padres de familia expresaron que los niños deben realizar actividades relacionadas con estas temáticas, además de las tareas que deben realizar como parte de sus actividades educativas (clases), estas temáticas son: lógicomatemáticas, lecto-escritura y habilidades sociales.

Además, se identificó que la herramienta a diseñar debía ser un juego, donde los niños no perciban que están realizando tareas de sus clases remotas. Sin embargo, incluir desafíos relacionados con las temáticas principales identificadas. Estos desafíos se clasificaron en tres etapas de acuerdo a la edad: etapa 1 (5-6) años, etapa 2 (7-8) años, etapa 3 (9-10) años. Con base en las necesidades detectadas y las propuestas de la etapa de Idear, se determinó que, para poder hacer uso de la herramienta, la persona adulta (padre de familia/tutor) debe registrarse en la herramienta y posteriormente registrar los datos de los niños que harán uso de la herramienta.

Otro resultado del diagrama de afinidad fue que los niños generalmente comparten sus dispositivos electrónicos, por lo que la herramienta debe permitir agregar a más de un niño por perfil del tutor. El adulto podrá personalizar el aprendizaje del niño dependiendo la etapa que corresponda (edad). En la personalización se determinan cuántos desafíos se realizarán al día o a la semana por cada temática. Así como también el adulto podrá monitorear el progreso de las habilidades desarrolladas del niño. La herramienta permitirá realizar juegos conformados por tres categorías:

- Actividad de aprendizaje: el niño obtiene algún tipo de información sobre la temática abordada antes de aplicar el desafío.

- Actividad digital: el niño hace uso de la aplicación para realizar una actividad relacionada con la temática.

- Actividad híbrida: el propósito es permitir la interacción del niño con el adulto y que ambos realicen alguna actividad sugerida por la herramienta en el que se priorice la actividad física.

Cada vez que el niño termina un desafío puede obtener una recompensa la cual consiste en elegir un sticker que podrá visualizarse con realidad aumentada. Se optó por esta tecnología para favorecer la movilidad del niño.

\section{Kids-InteracTOOL}

Kids-InteracTOOL es una herramienta para tableta digital, que tiene una interfaz amigable con el usuario; solo se presenta la información mínima necesaria y en caso de tener dudas sobre las actividades a realizar con la herramienta, se puede acceder a una

3 Liga de Figma sobre pantallas de la vista del adulto. https://www.figma.com/file/6ri8NnmqoslvQZ0BJhBwr2/MexIHC?node$\underline{\mathrm{id}=42 \% 3 \mathrm{~A} 5}$ guía o a los íconos de información (si son adultos), o bien a la repetición de instrucciones para los niños (audio y texto).

\subsection{Características}

La herramienta consiste en dos vistas principales: la vista del adulto $^{3}$ y la vista del niño ${ }^{4}$. Dependiendo de las vistas, es el alcance de acciones que puede realizar el usuario, así como el diseño que visualiza; para el adulto son colores más sobrios, mientras que para los niños son colores más llamativos.

Una de las características de la herramienta es promover la interacción entre el adulto y el niño mediante una actividad guiada, sencilla de realizar. Sin embargo, esta actividad, la cual forma parte de la actividad híbrida puede desarrollarse con cualquier otro participante. Adicionalmente, las actividades de los desafíos deben poder realizarse en periodos cortos de tiempo. Otra característica es que los participantes tienen una preferencia por el empleo de herramientas en plataformas móviles al ser más intuitivas y estar relacionados con su uso, por lo que el diseño se realizó precisamente para dispositivos móviles.

\subsubsection{Vista adulto}

La vista de adulto representa la vista de administrador, debido a que a través de su cuenta tiene permitido el realizar configuraciones sobre los perfiles de los niños (Figura 3 - a) y conocer el progreso del niño (Figura 3 - b).

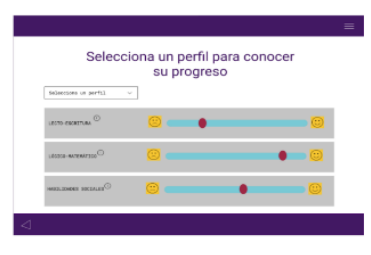

a. Pantalla de Progreso

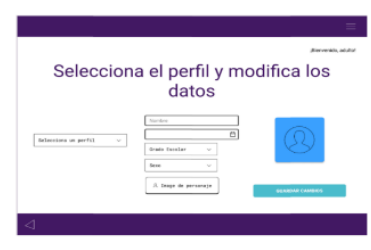

b. Pantalla de Perfil de datos del Infante
Figura 2. Pantallas Vista Adulto (1).

Adicionalmente, tiene la facultad para determinar si el niño seguirá una ruta de aprendizaje recomendado por el sistema en función de su edad (Figura 3 - a), o bien seleccionar la cantidad de desafíos de acuerdo a las categorías de desarrollo de habilidades disponibles (Figura 3 - b) .
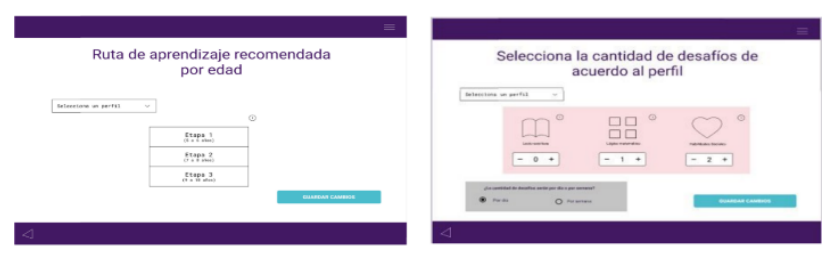

a. Pantalla de Ruta de aprendizaje Recomendada

b. Pantalla de Selección de desafíos por categoría

Figura 3. Pantallas Vista Adulto (2).

Por otro lado, Kids-InteracTOOL envía notificaciones al adulto para informar que el niño está por completar un desafío, y otra notificación para que valide que la actividad fue realizada con éxito, de manera tal que la última actividad de cada desafío incluye una actividad guiada en la que se fomenta la interacción entre el niño y el adulto (o algún otro individuo que pueda realizar la actividad en compañía del niño) la cual requiere un tiempo corto de participación, dado que durante las entrevistas se encontró que los papás tienen el deseo de realizar más actividades con sus niños,

\footnotetext{
${ }^{4}$ Liga de Figma sobre pantallas de la vista del niño. https://www.figma.com/file/6ri8NnmqoslvQZ0BJhBwr2/MexIHC?node-id=0\%3A1
} 
pero a veces el tiempo es una limitante (ver Figura de notificaciones).

\subsubsection{Vista Niño}

En esta vista, el niño lleva a cabo los desafíos y puede acceder a su librería de stickers (recompensas), los cuales obtiene con la finalización de los desafíos. También puede ingresar a la galería de fotos y videos que almacena de acuerdo a las actividades que le solicita el sistema que realice (actividad híbrida).

A continuación, se muestra el flujo de uno de los desafíos para la categoría de lógico-matemático, si bien el contenido de las actividades varía dependiendo de las temáticas (lecto-escritura ${ }^{5}$, lógico-matemático ${ }^{6}$, y habilidades sociales $\left.^{7}\right)$, su estructura permanece igual: se inicia con un mapa (Figura 4 - a), después una actividad de aprendizaje (Figura 4 - b), una actividad digital (Figura 4 - c) y una actividad híbrida (Figura 4 - d).

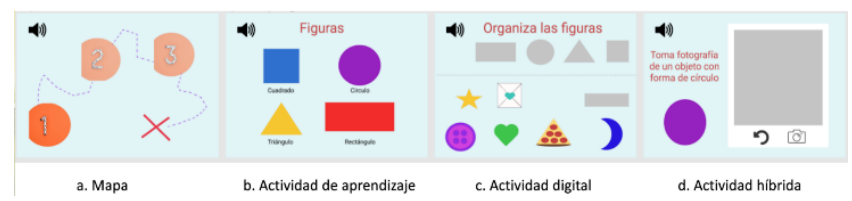

Figura 4. Pantallas Vista niño (1).

El otro elemento que compone la vista del niño es la librería de stickers (Figura 5 - a), puede seleccionar cualquiera de los stickers que ha adquirido (Figura 5 -b) y por medio de la cámara del dispositivo móvil puede visualizar al personaje con Realidad Aumentada (Figura 5 -d). También puede ver en la galería (Figura 5 -c) las fotografías y videos que se han almacenado en el sistema durante la realización de las actividades híbridas.

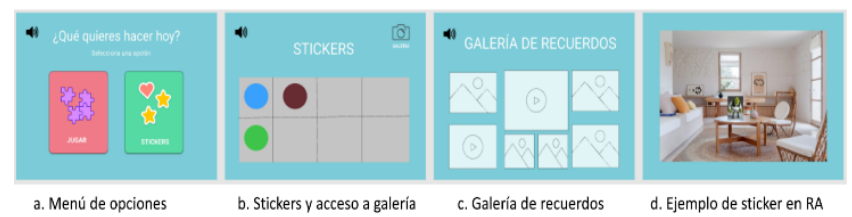

Figura 5. Pantallas Vista niño (2).

\section{Resultados de la sesión de Validación}

Respecto a los resultados del TAM, se obtuvo un puntaje promedio de 5.9 (máximo 7) en la dimensión de utilidad y un puntaje de 4.8 para la dimensión de facilidad de uso. La dimensión de Facilidad de uso tuvo un puntaje menor, lo cual está relacionado con que dos de las tres madres de familia no se sienten familiarizadas con el uso de la tecnología. Por otro lado, la Tabla 2 muestra los resultados de las siete dimensiones del GEQ por participante y el puntaje promedio. Se puede observar que $\mathrm{P} 2$ tiene un puntaje de 3 (máximo 4) en la dimensión de Afecto negativo, esto se debió a que P2 esperaba que el prototipo fuera funcional, pues quería ver y elegir los stickers, así como utilizar la función de Realidad Aumentada.

Tabla 2. Resultados de la Experiencia de Juego (GEQ)

\begin{tabular}{|c|c|c|c|c|}
\hline $\begin{array}{c}\text { Participante (Edad, } \\
\text { sexo)/ Dimensión }\end{array}$ & $\begin{array}{c}\text { P1 } \\
\text { (5 años, } \\
\text { M) }\end{array}$ & $\begin{array}{c}\text { P2 } \\
\text { (6 años, } \\
\text { F) }\end{array}$ & $\begin{array}{c}\text { P3 } \\
\text { (8 años, } \\
\text { M) }\end{array}$ & AVG \\
\hline Competencia & 4.0 & 2.9 & 4.0 & 3.33 \\
\hline
\end{tabular}

\footnotetext{
${ }^{5}$ Flujo del desafío lecto-escritura https://bit.ly/3uMuiuh
}

\begin{tabular}{|c|c|c|c|c|}
\hline Inmersión & 4.0 & 2.5 & 4.0 & 3.50 \\
\hline Flujo & 3.0 & 2.0 & 0 & 1.67 \\
\hline Tensión & 0 & 2.0 & 0 & 0.67 \\
\hline Desafío & 2.5 & 3.5 & 0 & 2.00 \\
\hline Afecto Negativo & 0 & 3.0 & 0 & 1.00 \\
\hline Afecto Positivo & 4.0 & 2.0 & 4.0 & 3.33 \\
\hline
\end{tabular}

A pesar de lo anterior, los resultados del GEQ muestran en promedio una experiencia de juego positiva en sus siete dimensiones; por ejemplo, los participantes estuvieron inmersos en el juego y este les brindó una influencia positiva.

\section{Conclusión}

De manera general, Kids-InteracTOOL recibió una aceptación positiva por parte de los niños participantes, lo que nos indica que existe interés por parte de los adultos y niños para usar este tipo de herramientas para apoyar el desarrollo de habilidades educativas y sociales fuera de las labores oficiales de la escuela. El proceso de diseño utilizado en este proyecto permitió al equipo de trabajo tener la experiencia de diseñar a partir de una problemática particular y haciendo uso de métodos de interacción humano-computadora para llegar a una posible solución.

\section{Agradecimientos}

Agradecemos el apoyo y disposición de las madres de familia que participaron en este proyecto, así como de los niños que proporcionaron sus comentarios para mejorar el diseño del prototipo.

\section{Referencias}

[1] Asociación Mexicana de Internet Mx.. (2021). $17^{\circ}$ Estudio sobre los Hábitos de los Usuarios de Internet en México 2021. octubre 01, 2021, de Asociación Mexicana de Internet $\mathrm{Mx}$.

[2] Brand, J.E. (2003). Don't criticize the effects of video games on kids, exploit them. On Line Opinion.(26 May).

[3] Ciudades Amigas de la Infancia. (2020). Se dispara el uso de pantallas en niños y niñas durante el confinamiento. octubre 1, 2021, de UNICEF Sitio web: https://ciudadesamigas.org/pantallas-infancia-cuarentena/

[4] Derboven, J. Zaman, B., Vissers, J., Geerts, D., \& De Grooff, D. The Fun-Serious Ambiguity in Educational Games.

[5] Educational Gaming Mobile App in Assisting Kids in Learning Math and English (Link:https://papers.ssrn.com/sol3/papers.cfm?abstract_id=3 714728)

[6] Griffiths, M.D.(2002). The educational benefits of videogames. Education and health, 20(3), 47-51.

[7] Instituto Mexicano del Seguro Social. (2017). Encuesta nacional de empleo y seguridad social. Instituto Nacional de Estadística, Geografía e Informática.)

\footnotetext{
${ }^{6}$ Flujo del desafío lógico-matemático https://bit.ly/3oFwant

${ }^{7}$ Flujo del desafío habilidades sociales https://bit.ly/3DfMD5V
} 
[8] Interaction Design Foundation. 2021. Personas. https://www.interaction-design.org/literature/topics/personas

[9] Juliet M Corbin and Anselm Strauss. 1990. Grounded theory research: Procedures, canons, and evaluative criteria. Qualitative sociology 13, 1 (1990), 3-21.

[10] Lee Skrypchuk Voula Gkatzidou, Joseph Giacomin. 2021. Automotive Human Centred Design Methods (1st. ed.). Boston: De Gruyter, Berlin, Chapter 12, 50-51. https://doi.org/10.1515/9783110677515-013

[11] López-Faican, L., \& Jaen, J. (2020). EmoFindAR: Evaluation of a mobile multiplayer augmented reality game for primary school children. Computers \& Education, 149, 103814.

[12] Reimers, F., Schleicher, A., Saavedra, J., \& Tuominen, S. (2020). Supporting the continuation of teaching and learning during the COVID-19 Pandemic. Oecd, 1(1), 1-38.

[13] Rocha, M., \& Dondio, P. (2021). Effects of a videogame in math performance and anxiety in primary school. International Journal of Serious Games, 8(3), 45-70.

[14] Shi, Y., Zhou, Q., Wen Tong, M., Cheng, Q., Xiao Cui, X., Cao, P., \& Fei Shi, Y. (2021). The Research Status and Development Trend of Educational Games Abroad in the Past Four Years. In 2021 The 7th International Conference on Frontiers of Educational Technologies (pp. 51-59). Association for Computing Machinery.
[15] Squire, K. D. (2008). Video games and education: Designing learning systems for an interactive age. Educational Technology, 17-26.

[16] The Lego foundation en apoyo de UNICEF. (2019) https://www.unicef.org/sites/default/files/2019-01/UNICEFLego-Foundation-Aprendizaje-a-traves-del-juego.pdf

[17] Thomas, J. M., \& DeRosier, M. E. (2010, June). Toward effective game-based social skills tutoring for children: An evaluation of a social adventure game. In Proc. of the Fifth FDG (pp. 217-223).

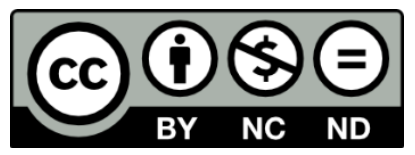

(C) 2021 by the authors. This work is licensed under the Creative Commons AttributionNonCommercial-NoDerivatives 4.0 International License. To view a copy of this license, visit http://creativecommons.org/licenses/by-nc-nd/4.0/ or send a letter to Creative Commons, PO Box 1866, Mountain View, CA 94042, USA. 